\title{
Mucocutaneous Occurrences of the Syphilis in Pregnant and HIV Positive Women
}

\section{Craina $\mathbf{M}^{1}$, Anca Bordianu²} Muresan $^{5}$

\author{
$\square^{3}$, Roxana Folescu4, Corina Matu ${ }^{4}$, Elena Bernad ${ }^{4}$, Izabella Petre ${ }^{1,4 *}$ and Anca
}

${ }^{1}$ Department of Obstetrics and Gynecology, University of Medicine and Pharmacy Timisoara, Romania

${ }^{2}$ Department of Plastic Surgery and Reconstructive Microsurgery, University of Medicine and Pharmacy, Bucharest, Romania

${ }^{3}$ Department of Morpho-Functional Sciences, Discipline of Histology, University of Medicine and Pharmacy lasi, Romania

${ }^{4}$ Department of Anatomy and Embryology, University of Medicine and Pharmacy Timisoara, Romania

${ }^{5}$ Department of Pathology, University of Medicine and Pharmacy Timisoara, Romania

\begin{abstract}
Introduction: "AIDS was the major epidemic of the $20^{\text {th }}$ century, and remains the epidemic of the $21^{\text {st }}$ century as well" thinks Prof. Jean-François Delfraissy, the director of the National Agency for Research on AIDS (ANRS). In the early 90's, over $50 \%$ of HIV-positive European children were living in Romania. Even today, most HIVIAIDS-positive people live in Romania - more than 7000 being children and adolescents. The purpose of my study is the correlation of the female mucocutaneous genital lesions with the clinical picture and the laboratory data, and the data correlation in pregnant women.
\end{abstract}

Matter and methods: The methodological support of the paper is represented by various materials obtained from official sources and personal research. The epidemiological analysis of the HIV infection cases was made based on data with sanitary-epidemiological indicators selected from annual records, in the time period from 2005 to 2010. To determine the eligibility criteria for developing the epidemic process, in the study were used classical methods of retrospective statistical analysis of the annual and multiannual cases. I have used a series of blood tests, of which the best known and most used are the ELISA and the Western-Blot tests. The reaction of Western-Blot is very much used in present to confirm or disprove the result of the ELISA test regarding HIV (test for specific antibodies), which sometimes can be false positive.

Results and discussions: It is interesting to note that the infected mother's antibodies can be transmitted to the fetus and it may persist in the fetus's blood up to 18 months. A test performed during this period is positive, meaning it would indicate the presence of the infection although it is possible that the fetus was not infected (46 cases). Therefore, a positive HIV test is not significant, only after the age of 18 months. Of the total of 47 pregnant women under observation 32 of them had undergone caesarean section (the other ones quitted our service). The age of the pregnant women was between 21 and 36 years. Gestational age was, except for 2 cases (39 weeks), between 34-36 weeks. Female newborns weighed between $2100 \mathrm{~g}-3300 \mathrm{~g}$, Al (Apgar Index) 8 or 9 . We also detected injuries caused by syphilis in 8 of our cases.

Conclusions: The genital chancre (genital ulceration) caused by syphilis makes a person more vulnerable to sexually transmitting or acquiring the HIV infection. The existence of syphilis increases the risk of acquiring HIV by 2 to 5 times.

Keywords: Mucocutaneous lesions; Syphilis; HIV

\section{Introduction}

According to European reports, the most common sexually transmitted diseases (STDs) are Chlamydia trachomatis infections, gonorrhea and syphilis. Compared to other European countries, the studies conducted by the European authorities in communicable disease epidemiology, show that Romania ranks first in frequency of syphilis cases reported [1,2].

Here, compared to other European countries, the syphilis incidence (number of cases/year) has increased since 1989 [3,4]. Even if the number of syphilis cases is still high, the tendency is to decrease the incidence of this disease - despite the high number of syphilis cases, Romania reported one of the highest rates of reduction in the number of syphilis cases (over 30\% since 2006).

In Romania, AIDS represents a syndrome associated with a marked disorder of the baby's body immunity. Currently, AIDS is the eighth cause of death in children aged 1-4 years, and sixth in young people aged 15-24 years.

\section{Material and Methods}

The methodological support of the paper is represented by various materials obtained from official sources and personal research. The epidemiological analysis of the HIV infection cases was made based on data with sanitary-epidemiological indicators selected from annual records, in the time period from 2005 to 2010. To determine the eligibility criteria for developing the epidemic process, in the study were used classical methods of retrospective statistical analysis of the annual and multiannual cases. I have used a series of blood tests, of

${ }^{*}$ Corresponding author: Petre Izabella, Department of Anatomy and Embryology, University of Medicine and Pharmacy Timisoara, EftimieMurgu 1-2, Timisoara, Romania, Tel: +40722646058; E-mail: dr.petreizabella@yahoo.com

Received February 13, 2014; Accepted April 28, 2014; Published May 10, 2014

Citation: Craina M, Bordianu A, Zamfir CL, Folescu R, Matu C, et al. (2014) Mucocutaneous Occurrences of the Syphilis in Pregnant and HIV Positive Women. J AIDS Clin Res 5: 306. doi:10.4172/2155-6113.1000306

Copyright: (C) 2014 Craina M, et al. This is an open-access article distributed under the terms of the Creative Commons Attribution License, which permits unrestricted use, distribution, and reproduction in any medium, provided the original author and source are credited. 
which the best known and most used are the ELISA and the WesternBlot tests. The reaction of Western-Blot is very much used in present to confirm or disprove the result of the ELISA test regarding HIV (test for specific antibodies), which sometimes can be false positive.

A test performed during this period is positive, meaning it would indicate the presence of the infection although it is possible that the fetus was not infected ( 46 cases). Therefore, a positive HIV test is not significant, only after the age of 18 months. Of the total of 47 pregnant women under observation 32 of them had undergone caesarean section (the other ones quitted our service). The age of the pregnant women was between 21 and 36 years. Gestational age was, except for 2 cases (39 weeks), between 34-36 weeks. Female newborns weighed between 2100 g-3300 g, AI (Apgar Index) 8 or 9 . We also detected injuries caused by syphilis in 8 of our cases.

Diagnosis is possible with the use of two types of serologic tests: 1) nontreponemal tests (e.g., Venereal Disease Research Laboratory [VDRL] and RPR) and 2) treponemal tests (e.g., fluorescent treponemal antibody absorbed [FTA-ABS] and T. pallidum particle agglutination [TP-PA]). The use of only one type of serologic test is insufficient for diagnosis because false-positive nontreponemal test results are sometimes associated with various medical conditions unrelated to syphilis.

\section{Results and Discussions}

Regarding the distribution of cases, according to HIV staging, clinical and paraclinical staging is represented in the chart below:

- 1 case was diagnosed in stage I, which represents $2.1 \%$;

- 4 cases were diagnosed in stage II, which represents $8.5 \%$;

- 27 cases were diagnosed in stage III, which represents 57.4\%;

- 8 cases were diagnosed in stage IV, which represents $17 \%$;

- 7 cases were diagnosed in stage $\mathrm{V}$, which represents $15 \%$.

Out of the total, the patients who presented lesions caused by syphilis were classified in stage I (1 case), stage II (3 cases) and stage III (4 cases).

Syphilis may present with protean manifestations, but most commonly manifests as genital ulcerative disease during the primary stage and as rash, fever and adenopathy during the secondary stage. During the secondary stage, dissemination of treponemes throughout the body occurs affecting both mucocutaneous and visceral sites. Although liver involvement due to syphilis has been recognized in the general population for over 400 years [5], published reports are sparse, with the largest series in the recent literature consisting of only 17 cases [6].

Genital chancre (genital ulcer) caused by syphilis makes a person more vulnerable to convey, namely to acquire HIV infection sexually. The risk of acquiring HIV in the conditions of existence of syphilis is 2-5 times higher.

Ulcerative STDs that cause chancres, ulcers or skin or mucous membranes cracking, such as syphilis, destroy the barriers against infection. Genital ulcers caused by syphilis bleed easily, so contact with oral or rectal mucosa during sex, increase susceptibility to HIV (Figure 1-5).

The presence of other SDTs is also a risk factor associated with HIV transmission. All women should be screened serologically for

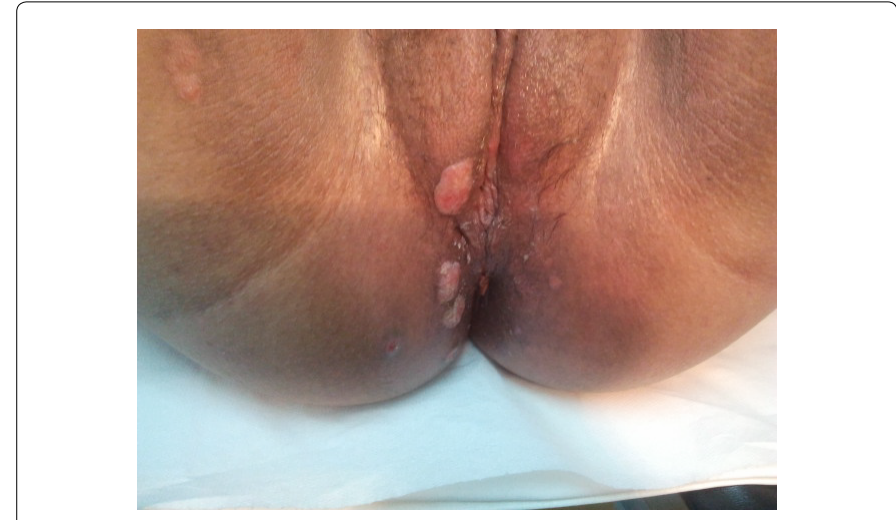

Figure 1: Pregnancy in 14 weeks - syphilis lesions in the anal region and intergluteal.

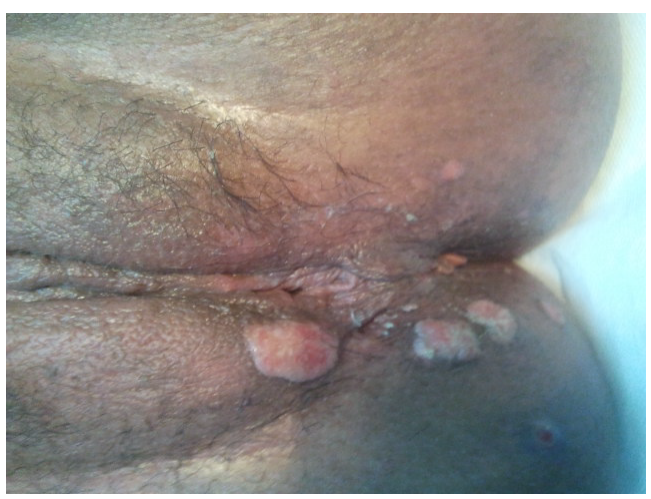

Figure 2: Pregnancy in 12 weeks - syphilis lesions in lip and vaginal introital region.

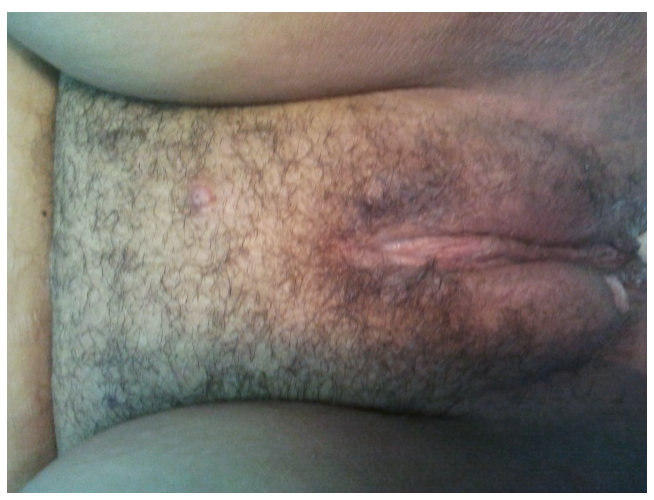

Figure 3: Pregnancy in 7 weeks - syphilis lesions in suprapubic and perilabial region.

syphilis during the early stages of pregnancy. The majority of states mandate screening at the first prenatal visit for all women. Antepartum screening by nontreponemal antibody testing is typical, but in some settings, treponemal antibody testing is being used. Pregnant women with reactive treponemal screening tests should have confirmatory testing with nontreponemal tests with titers [7].

Persons with a positive treponemal screening test should have a standard nontreponemal test with titer to guide patient management decisions. If the nontreponemal test is negative, then a different 


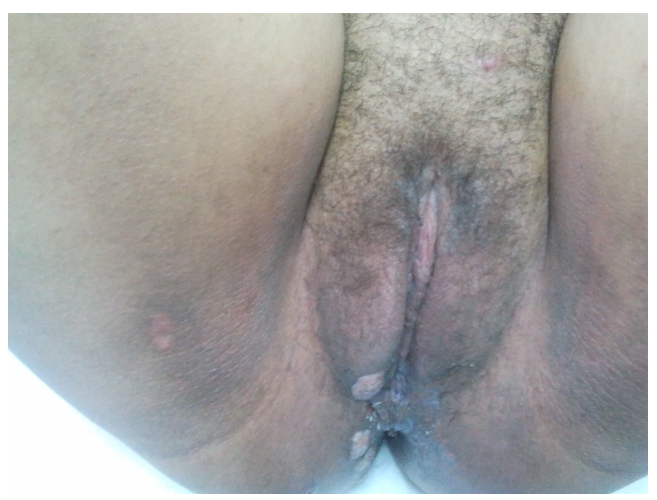

Figure 4: Pregnancy in 24 weeks - syphilis lesions in perianal and lower labial region.

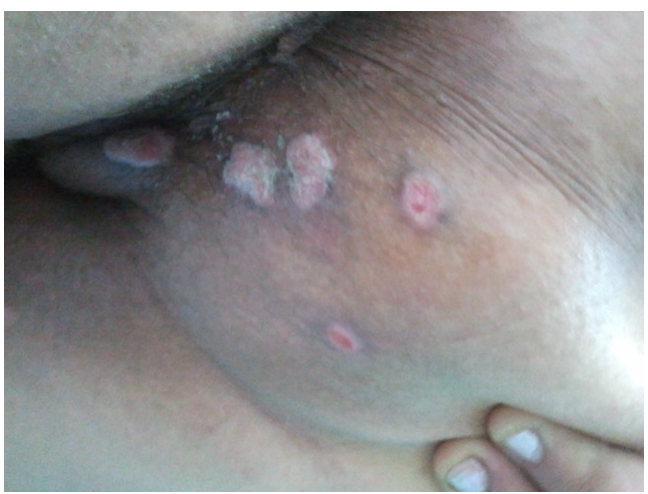

Figure 5: Pregnancy in 17 weeks - syphilis lesions in gluteal and intergluteal region.

treponemal test should be performed to confirm the results of the initial test. If a second trepomenal test is positive, treatment decisions should be discussed in consultation with a specialist. However, for the majority of HIV-infected patients, serologic tests are accurate and reliable for the diagnosis of syphilis and for following the response to treatment.

Syphilitic sore can increase the risk of HIV transmission by disrupting mucosal and epithelial barriers [8] and is followed several weeks or months later by widespread cutaneous, mucosal, and sometimes systemic indications of the dissemination of the spirochetes of secondary syphilis.

In the USA, the rate of HIV transmission from untreated mothers to fetuses/newborns is about $25 \%[9,10]$.

Studies in several countries show a variable proportion of transmission rate with a minimum of $12.9 \%$ in a multicenter European study and a maximum of $45-48 \%$ in Nairobi, Kenya, and other cities in developing countries [11-13].

Like the work of Wolff $\mathrm{T}$ et al. unusual serologic responses have been observed among HIV-infected persons who have syphilis. The majority of reports have involved serologic titers that were higher than expected, but false-negative serologic test results and delayed appearance of seroreactivity also have been reported. However, unusual serologic responses are uncommon, and the majority of specialists believe that both treponemal and nontreponemal serologic tests for syphilis can be interpreted in the usual manner for the majority of patients who are coinfected with T. pallidum and HIV [14].

\section{Conclusions}

It is important to be realize that no community/country is not completely immune to HIV, which is why instead of isolating the infected persons, it is mandatory to educate the population, especially the young people, who are at increased risk for certain behaviors, such as intravenous drug abuse, homosexuality and multiple sex partners. Early detection of the infection is the best method to control the spread of the virus in the community. Psychological and social aspects of the HIV infection have been recognized since its identification as a STD. Unfortunately, even after more than 25 years since identifying the condition, people carrying HIV face social and cultural discrimination, especially in poor countries where the infection reached epidemic proportions. Although they need the support of the community and their families to accept and cope with the disease, the HIV infected people, especially the pregnant women, are often emotionally and psychologically abused by others.

The postnatal transmission of HIV from mother to child has been clearly documented, involving the strong colostrum and breast milk as vehicles of infection; the virus can be isolated from both fluids. In rare cases, the mothers were infecting through transfusions performed after birth and transmitted the infection to their children, the maternal nutrition being the only risk factor. In developing countries, this is an important way of HIV transmission, especially where mothers continue to breast feed for a longer period than what is considered to be the usual in developed countries. A meta-analysis of several prospective studies indicates that the risk of breast feeding allocation was $7-22 \%$.

\section{References}

1. World Health Organization (2011) Towards eliminating congenital syphilis. Progress Report, WHO.

2. World Health Organization (2012) Sexually transmitted infections. Regional Office for Europe, WHO.

3. Tridapalli E, Capretti MG, Sambri V, Marangoni A, Moroni A, et al. (2007) Prenatal syphilis infection is a possible cause of preterm delivery among immigrant women from eastern Europe. Sex Transm Infect 83: 102-105.

4. Hawkes S, Matin N, Broutet N, Low N (2011) Effectiveness of interventions to improve screening for syphilis in pregnancy: a systematic review and metaanalysis. Lancet Infect Dis 11: 684-691.

5. Schlossberg D (1987) Syphilitic hepatitis: a case report and review of the literature. Am J Gastroenterol 82: 552-553.

6. Crum-Cianflone N, Weekes J, Bavaro M (2009) Syphilitic hepatitis among HIVinfected patients. Int J STD AIDS 20: 278-284.

7. Sheffield JS, Sánchez PJ, Morris G, Maberry M, Zeray F, et al. (2002) Congenital syphilis after maternal treatment for syphilis during pregnancy. Am J Obstet Gynecol 186: 569-573.

8. Greenblatt RM, Lukehart SA, Plummer FA, Quinn TC, Critchlow CW, et al (1988) Genital ulceration as a risk factor for human immunodeficiency virus infection. AIDS 2: 47-50.

9. ACOG Committee on Obstetric Practice (2004) ACOG committee opinion number 304, November 2004. Prenatal and perinatal human immunodeficiency virus testing: expanded recommendations. Obstet Gynecol 104: 1119-1124.

10. American College of Obstetricians and Gynecologists (2003) ACOG Committee Opinion. Primary and preventive care: periodic assessments. Obstet Gynecol 102: $1117-1124$

11. Athe MN, Tsibris Martin S, Hirsch (2010) Antiretroviral Therapy for Human Immunodeficiency Virus Infection. In Mandell GL, Bennett JE, Dolin R (Eds.) Bennett's Principles and Practice of Infectious Diseases. (7thedn), Churchill Livingstone, Elsevier: 1847-1848.

12. Geoffrey A Weinberg, George K Siberry (2010) Pediatric Human Immunodeficiency Virus. In Mandell GL, Bennett JE, Dolin R (Eds.), Bennett's Principles and Practice of Infectious Diseases. (7th Edn), Churchill Livingstone Elsevier: 1818-1819. 
Citation: Craina M, Bordianu A, Zamfir CL, Folescu R, Matu C, et al. (2014) Mucocutaneous Occurrences of the Syphilis in Pregnant and HIV Positive Women. J AIDS Clin Res 5: 306. doi:10.4172/2155-6113.1000306

Page 4 of 4

13. Marvin S Reitz, Robert C Gallo (2010) Human Immunodeficiency Viruses. n Mandell GL, Bennett JE, Dolin R (Eds.), Bennett's Principles and Practice of Infectious Diseases. (7thedn), Churchill Livingstone, Elsevier: 2323-2333.
14. Wolff T, Shelton E, Sessions C, Miller T (2009) Screening for syphilis infection in pregnant women: evidence for the U.S. Preventive Services Task Force reaffirmation recommendation statement. Ann Intern Med 150: 710-716. 